\title{
Extensive type IIIB bone loss of the acetabulum and suitability of 3D printings during planning
}

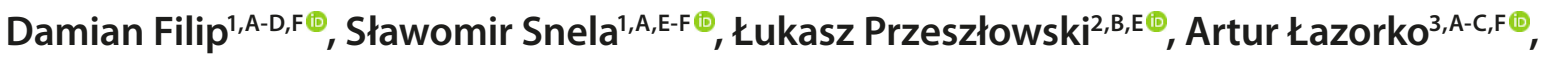 \\ Weronika Dziura ${ }^{2, A-B, D \odot}$ \\ ${ }^{1}$ Department of Orthopedics and Traumatology University of Rzeszów, Institute of Medical Sciences, Rzeszów, Poland \\ 2 University of Technology, Rzeszów, Poland \\ ${ }^{3}$ Department of Orthopedics and Traumatology, New Medical Techniques Specialist Hospital of the Holy Family, \\ Rzeszów, Poland \\ A - Research concept and design, B - Collection and/or assembly of data, C - Data analysis and interpretation, \\ $D$ - Writing the article, E-Critical revision of the article, $F$ - Final approval of article
}

Filip DF, Snela S, Przeszłowski Ł, Łazorko A, Dziura W. Extensive type IIIB bone loss of the acetabulum and suitability of 3D printings during planning. J Pre-Clin Clin Res. 2021; 15(2): 63-67. doi: 10.26444/jpccr/135744

\begin{abstract}
Introduction. Despite the advances made in medicine, arthroplasty performed in young patients is still associated with an increased risk of complications. Massive destruction of the acetabular ring is a challenge, requires careful planning, and the use of various surgical techniques and implants.

Case Report. The case is presented of a young woman with a Paprosky type IIIB defect of the acetabular rin who underwent a revision total hip arthroplasty. During planning, 3D printed bone models were used because the choice of surgical technique was not obvious. The 3D prints, together with the radiological diagnostics performed, provided a real picture of the problem, and a better understanding of the pathoanatomy.

Conclusions. Multiple, unpunished trialing on printed models by using different trial implants make it possible to test the feasibility and practice the techniques. It may prevent making the mistake of choosing wrong the surgical technique. Additionally, the models give better visualization of the patient's anatomy during the surgery lifetime.
\end{abstract}

\section{Key words}

3D printing, revision, joint, reconstructive surgical procedures

\section{INTRODUCTION}

Primary total hip arthroplasty (THR) is frequently described as one of the greatest advances in medicine of the 20th century [1]. More and more THR surgeries are undertaken which, on the one hand, illustrates the growing needs of society, and on the other hand underlines the effectiveness of this method of surgical treatment. The results of primary arthroplasty vary, although their 15-year 'survival' period is considered to be 'good' and 'very good'. However, THR performed in young patients is still associated with an increased risk of complications [2].

The number of revision hip arthroplasty varies between $10 \%-$ $20 \%$ of the number of primary arthroplasty [2]. According to the data of the Norwegian Arthroplasty Registry, aseptic loosening accounts for $37 \%$ of revisions [3]. Some publications indicate an even higher percentage of revision operations due to aseptic loosening - close to $63 \%[4,5]$. Revisions are clearly more often caused by loosening of the acetabular cup rather than the femoral stem [3]. Depending on the time from the first symptoms of loosening and the usually complex pathomechanism of this process, various defects of the acetabulum and/or of the proximal end of the femur are found [4]. The term 'aseptic loosening' means mechanical damage to the prosthetic-bone interface as a result of a focal, periprosthetic inflammatory reaction caused by the precipitation of implant particles [5]. In the

Address for correspondence: Damian Filip, Institute of Medical Sciences, Department of Orthopedics and Traumatology, University of Rzeszów, Poland E-mail: damian.a.filip@gmail.com

Received: 21.03.2021; accepted: 13.04.2021; first published: 29.04.2021 first stage, particles from the prosthesis articulation surfaces are deposited in the joint and the surrounding tissues. They trigger a focal inflammatory process in which various cells of the immune response are involved, including fibroblasts, macrophages, lymphocytes, neutrophils and osteoclasts. The type of immune cellular response, however, depends on the origin, shape and size of the implant particles. Once initiated, the processes of the immune response lead to the loosening and migration of the implant, destruction of the bone tissue around it, and ultimately to worsening discomfort, pain and gait failure of the patient $[4,5]$. Acetabular and femoral defects are classified according to the commonly accepted Paprosky classification and vary from the minor (type I) to extensive destruction (types III and IV) $[6,7,8,9]$.

The aim of hip revision surgery is at least to regain or improve the quality of life and gait function that the patient presented after the primary prosthesis, and to eliminate pain and discomfort $[10,11,12]$. Preserving as much of the remaining acetabular bone stock as possible is important for any revision procedure, but it is especially essential in young patients who are likely to experience a few more revisions during their lifetime. Massive destruction of the acetabular ring is a challenge and require especially careful planning, and often the use of bone grafts and various implants. When planning a revision procedure, the operator must have a backup plan and associated implants in place. There are many possibilities for using implants in the most complex reconstruction cases. Traditionally, larger cavities in the acetabulum, both in primary and revision procedures, were reconstructed using compacted bone grafts and a cemented cup. Currently, there are other options for treating massive 
destruction of the acetabular ring, such as revision meshes and baskets, revision shells with or without a revision basket, structural bone grafts, highly porous titanium augment, and custom made implants $[7,10]$. The case is presented of a young woman with an extensive type IIIB defect of the acetabular ring, a type II defect of the proximal femur, and a fracture of the greater trochanter. She successfully underwent a revision total hip with the G7 OsseoTi Multi Hole revision shell with screws, Regenerex augment, Wagner SL revision stem, compacted allogeneic bone grafts, and absorbing calcium sulfate with vancomycin and gentamicin (Stimulan). The use of standard revision implants was not an obvious solution due to the extent of the defect, bone quality and the young age of the patient. Three-dimensional (3D) printed models, based on precise anatomy derived from CT, proved to be a very helpful tool for understanding the pathoanatomy, better plan a surgical technique and practice it with impunity [13].

\section{CASE REPORT}

A 31-year-old patient was admitted to our Department in December 2019 due to severe pain in the hip joint, difficulty in movement, with the symptoms of jumping in the hip joint, radiolucent changes surrounding the acetabular and femoral implants, and damage to the polyethylene liner and the acetabular shell (Fig. 1).

In childhood, she was treated due to congenital dislocation of the left hip joint with necrosis of the femoral head. Overhead hip traction and later immobilisation with cast was employed. At the age of 12.5 years, due to the residual dysplasia of the left hip with features of a history of femur head necrosis and varus hip, a transiliacal pelvic osteotomy was performed and she was provided with a hip cast. At the age of 18 , she underwent another surgery due to walking failure. The greater trochanter was distalized and stabilized with screws (Fig. 2). The implants were removed two years later.

At the age of 22, she was re-operated on due to increasing hip pain at rest, significant limitation in the range of hip movements, difficulty in moving, and intensification of radiological features of degenerative changes. From the Hardinge lateral approach, a $50 \mathrm{~mm}$ Plasmacup acetabular shell with 2 screws, a Plasmacup SC 28 PE asymmetric polyethylene liner, a $28 \mathrm{~mm}$ metal head and a modular Metha stem (Chifa Aesculap) were implanted. From 2011 - 2016, the patient was regularly monitored. During the visits, she did not report any significant pain, the range of hip movements was good, and the prosthesis components were fully integrated.

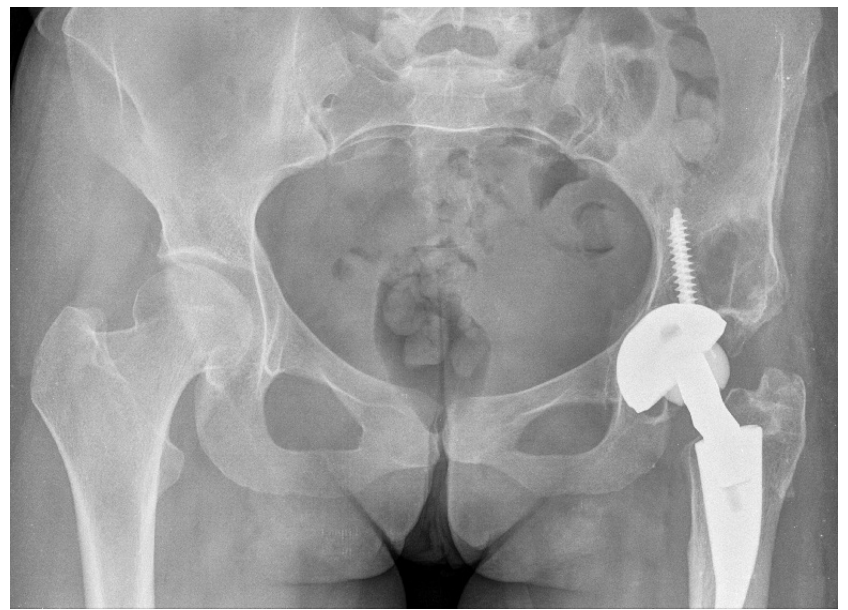

Figure 3. X-ray from 24.04.2019

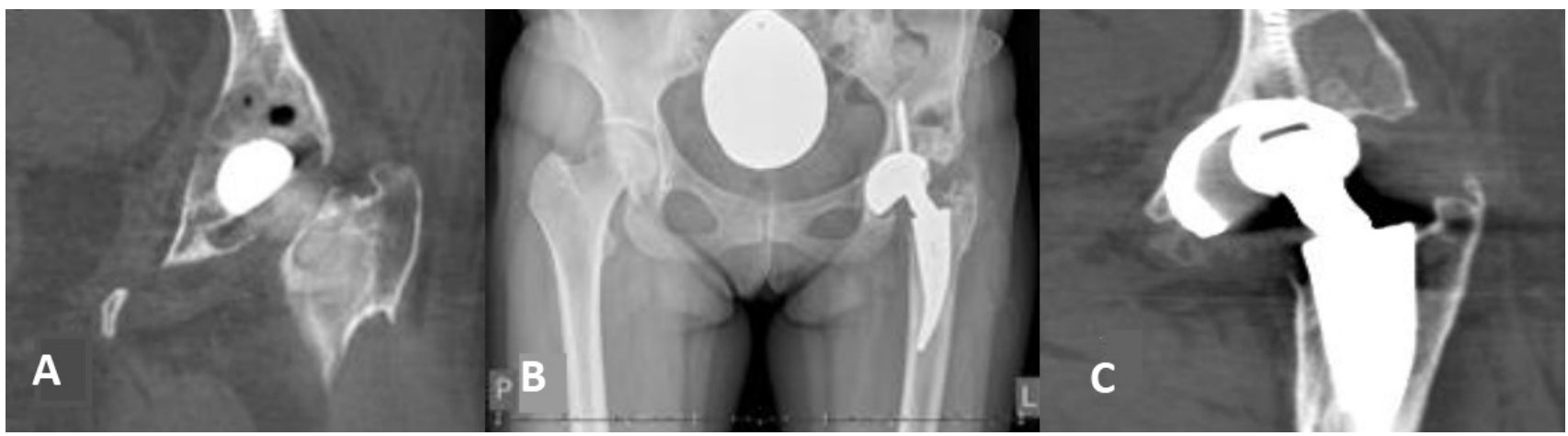

Figure 1. A-C. X-ray and CT images of the pelvis with loose parts of the prosthesis, bone defect of the acetabulum and femur and damage to the polyethylene liner and the acetabular shell
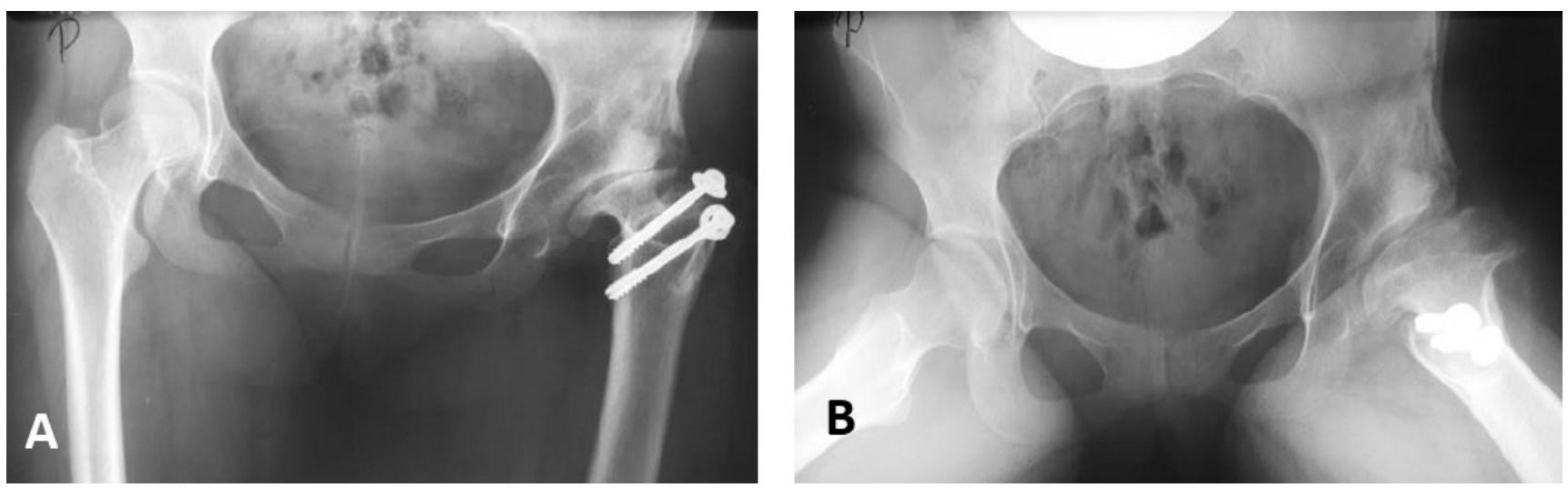

Figure 2. A-B. X-rays after distalization of the greater trochanter 
From February 2016 - April 2019, there was no documented control visit at the hospital clinic. During an examination in April 2019, she reported that she felt well during this period, became pregnant and gave birth. She reported for a check-up due to minor pain (especially after gym exercise and dancing) and a periodic feeling of 'blocking' of the hip (Fig. 3). At the next visit in October 2019, she reported severity of hip pain, the feeling of jumping, intensification of 'blocking', and difficulties in independent movement. The bone scintigraphy revealed aseptic, partial loosening of the acetabulum and femur implants. X-rays and CT displayed images of destruction in polyethylene liner, acetabulum shell and loosening of the implants (Fig. 1). Revision arthroplasty was performed on 16 December 2019. During the procedure, from the lateral approach, extensive bone defects and pseudotumoral lesions were found almost surrounding the entire acetabulum and stem. The polyethylene liner was completely destroyed and present only in several fragments. Significant loss of the acetabulum shell in the upper pole and almost completely damaged screw heads were seen. There were no macroscopic features of infection. The intraoperative microbiological test result was ambiguous. All loose and destroyed prosthesis components and some of pathologic tissue were removed. However, the weakened trochanter major was broken during manipulation of the retractors. Due to extensive bone loss, blackening of all tissues that prevented their safe identification, lack of suitable instruments and implants, and the operating team not sufficiently experienced, a temporary cement spacer $(50 \mathrm{~mm}$ in diameter with long stem - Aesculap) was implanted (Fig. 4). The patient was discharged from the department in good general and local condition.

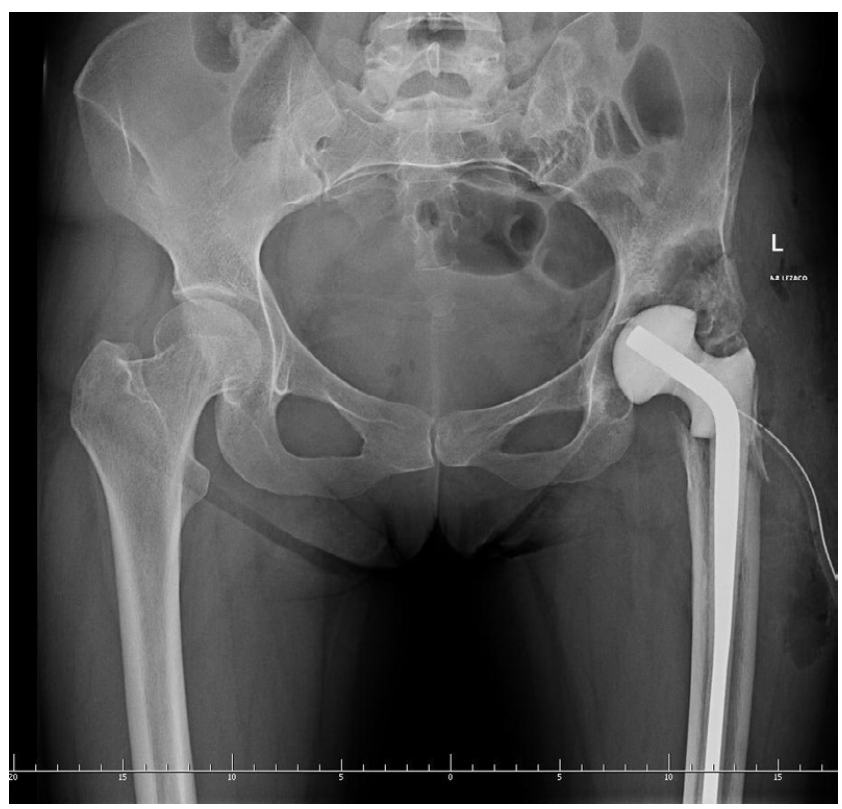

Figure 4. X-ray from 17.12.2019

Preparations for the next step lasted three months. Several possible solutions were considered [7]: 1. Structural allograft from the femoral head to restore the acetabular roof. Compacted allogeneic cancellous bone grafts to the bottom of the acetabulum and revision cup with screws. 2. Augment of porous titanium as a supplement to the acetabular roof, compacted allogeneic cancellous bone grafts for the bottom of the acetabulum and revision cup with screws. 3. Custommade implants. 4. Mutars Lumic prosthesis by Implantcast. 5. The 'cup-cage' technique. During planning, 3D printed bone models were used. Models were made at the University of Technology in Rzeszów.

Process of preparing and printing a 3D bone model in 1:1 scale. The medical model was created in the process of reconstructing the $2 \mathrm{D}$ dataset obtained from computed tomography. A volumetric model was generated, the correct area was separate, and the imaging artifacts related to the presence of the spacer were removed. Carryin-out appropriate modifications allowed obtaining a reconstruction of a key part of the pelvic bone (Fig. 5).

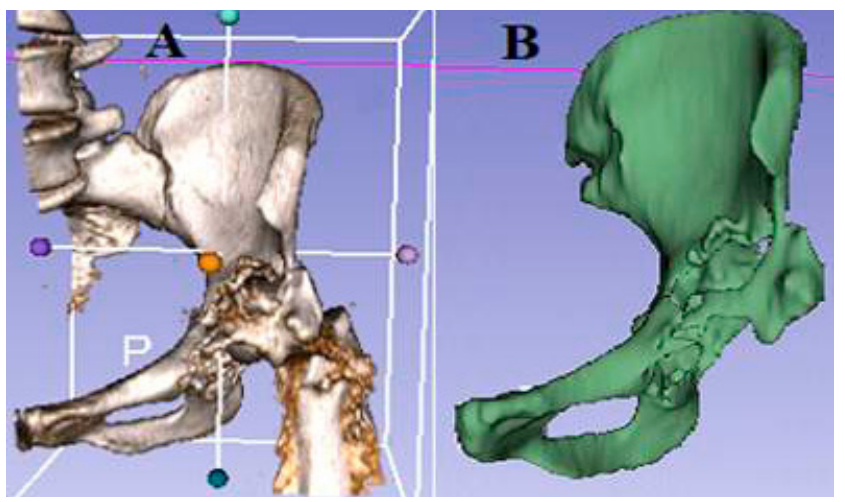

Figure 5. A-B. Screenshots from the 3D Slicer programme. A - Separate volumetric model of the pelvic bone with artifact. B-Modified medical model without artifacts

The finished medical modelwais divided into three parts. The printouts were made in FDM (Fused Deposition Modeling) technology, that is, as a result of the deposition of subsequent layers of melted thermoplastic PLA (Polylactide). The layer height was set at $0.2 \mathrm{~mm}$, thanks to which a high quality of the print surface was obtained. It was decided to divide the model into three parts due to the number of details (Fig. 6). Each part of the 3D model was printed for a total of about 12-14 hours. If it had been printed in one piece, the process would have taken about 18 hours. Moreover, there would be more supports, the removal of which could easily damage the model $[14,15]$.

After analyzing the performed diagnostic tests, trialing on $3 \mathrm{D}$ printed bone models and consultation with the revision specialists, the patient underwent the operation on $24 \mathrm{March}$ 2020, with an extended proximal lateral approach (Fig 7,8). The cement SPACER was removed. After cleaning the bottom of the acetabulum and gently reaming, the bottom was filled with compacted bone allografts and a small amount of Stimulan with vancomycin and gentamycin. The next step was to implant a Regenerex augment size 52 S (ZimmerBiomet), with additional stabilization using two titanium screws with a diameter of $6.5 \mathrm{~mm}$. At a later stage of the procedure, the G7 Osseo Ti $60 \mathrm{~mm}$ (Zimmer-Biomet) revision cup was implanted and additionally stabilized with eight screws. Between the cup and the augment, a small amount of bone cement was added. An asymmetric polyethylene liner with vitamin E was subsequently implanted. Next, after careful gradual reaming of the medullary canal of the femur, a Wagner SL revision stem (Zimmer-Biomet) was implanted. The last step was the implantation of a ceramic head with a diameter of $36 \mathrm{~mm}$. The remaining amount of Stimulan 

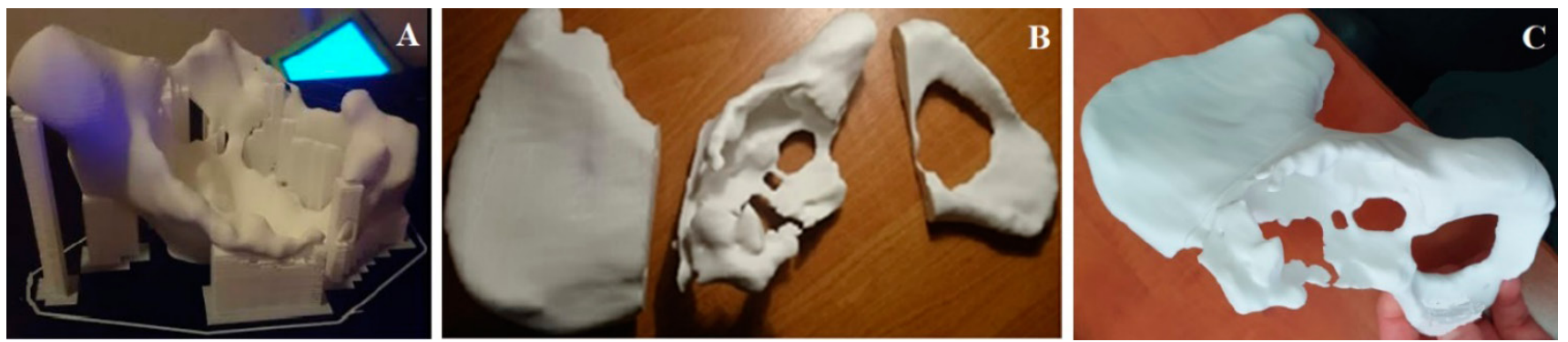

Figure 6. A-C, 3D prints of the pelvic bones. A - 1 of 3 parts with supports. B -3 parts without supports. $C-3$ parts connected together

was then applied to the joint. After reposition, satisfactory head coverage, primary implants stability and a good range of motion were obtained (Fig. 9A).

On the first day after surgery, the process of rehabilitation was started. For the first six weeks this involved walking while unloading the leg using crutches, and performing isometric exercises. During the follow-up visit, X-rays were taken (Fig. 9B), after which walking with partial loading of the limb with crutches was practiced. After about three months, the patient began to walk with full weight bearing without orthopedic appliances. The patient felt well and did not report any significant symptoms. Currently, she continues the motor improvement process and the development of gluteus muscles strength under the guidance of a physiotherapist. $\mathrm{X}$-rays taken after three months and one year show a well-integrated acetabular and femoral component of the prosthesis (Fig. 9C-E).

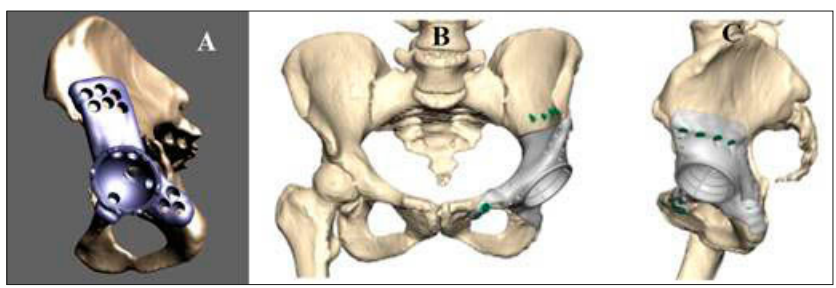

Figure 7. A-C. Custom-made implants: A. Triflange proposal (Zimmer-Biomet) B-C. Mutars Epore proposal (Implancast).
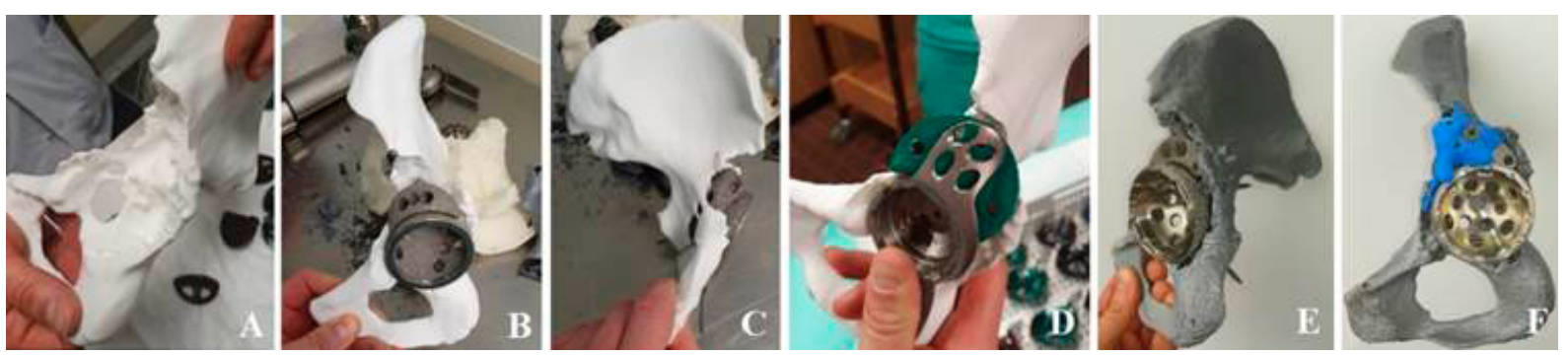

Figure 8. A-F. Preoperative planning
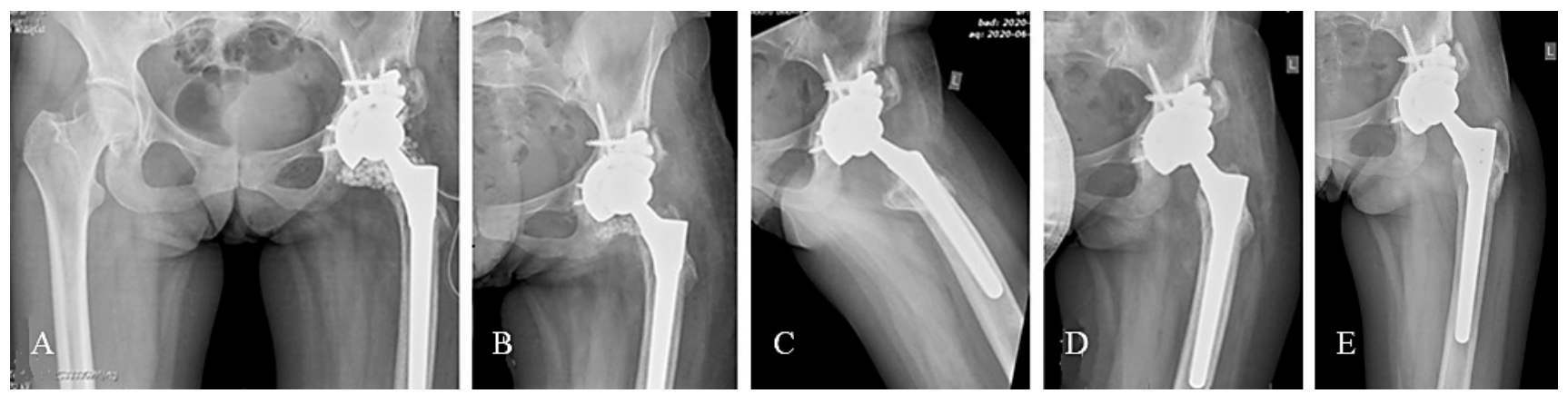

Figure 9. A-D. Postoperative X-rays: A. 1 day post-op B. 6 weeks post-op C-D. 3 months post-op. E. 1 year post-op. 
Years and the beginning of the Covid-19 pandemic. During this time, many necessary diagnostic tests were performed, and numerous consultations with revision specialists and orthopedic companies (custom-made implants) took place. Three-dimensional printed bone models on a 1:1 scale, based on precise anatomy derived from $\mathrm{CT}$, provided a real picture of the problem that had to be dealt with, and at the same time made it possible to re-dimension them multiple times with impunity. A method had to be selected that would save as much as possible of the remaining bone structure due to the numerous previous surgeries performed, and the patient's young age. After trialing the standard trial elements on the 3D models, a quite stable structure was obtained which ultimately allowed the selection of the most suitable method (Fig. 8,9). 3D printed bone models, together with the radiological diagnostics performed, provide a much greater idea of the problem than the radiological diagnostics alone. Moreover, the models gave a better visualization of the current patient anatomy during the operation lifetime.

The use of 3D models are recommended during the planning of preoperative technically demanding surgical procedures, especially for less experienced surgeons. The models provide the opportunity to practice the selected surgical technique, thereby avoiding errors many cases in selecting the wrong technique and implant. Despite the above advantages, the presented printing technique and the material used to print the $3 \mathrm{D}$ models have their limitations, especially dividing the model into three parts, the printing time for each of them was quite long. Each part was printed with supports, the removal of which could easily have damaged the model. Moreover, during reaming, the thermoplastic material began to melt and had the properties of a viscous fluid due to the high temperature generated. This caused the reamer to clog quickly. Additionally, the reamers were soiled with molten material, which is difficult but possible to remove. Therefore, the authors are currently testing other printers and materials with the aim of improving the printing process itself and, at the same time, allow easier work with the finished print.

\section{REFERENCES}

1.Zagra L. Advances in hip arthroplasty surgery: what is justified? EFORT Open Rev. 2017; 2: 171-178. doi: 10.1302/2058-5241.2.170008

2. Evans JT, Evans JP, Walker RW, Blom AW, Whitehouse MR, Sayers A. How long does a hip replacement last? A systematic review and metaanalysis of case series and national registry reports with more than 15 years of follow-up. Lancet. 2019; 393(10172): 647-654. doi: 10.1016/ S0140-6736(18)31665-9

3. Norwegian Arthroplasty Register, Total Hip Replacement. 2018: 9-59.

4. Gallo J, Vaculova J, Goodman SB, Konttinen YT, Thyssen JP. Contributions of human tissue analysis to understanding the mechanisms of loosening and osteolysis in total hip replacement. Acta Biomater. 2014; 10(6): 2354-2366. doi: 10.1016/j.actbio.2014.02.003

5. Wooley PH. How Has the Introduction of New Bearing Surfaces Altered the Biological Reactions to Byproducts of Wear and Modularity?. Clin Orthop Relat Res. 2014; 472(12): 3699-3708. doi: 10.1007/s11999-0143725-4

6. Telleria JJM, Gee AO. Classifications in brief: Paprosky classification of Acetabular bone loss. Clin Orthop Relat Res. 2013; 471(11): 3725-30. doi: 10.1007/s11999-013-3264-4

7. Mancino F, Cacciola G, Di Matteo V, et al. Reconstruction options and outcomes for acetabular bone loss in revision hip arthroplasty. Orthop Rev (Pavia). 2020; 12(Suppl 1): 8655. https://doi.org/10.4081/ or. 2020.8655

8. Niedźwiedzki Ł, Niedźwiedzki T. Reconstruction of massive acetabular bone loss with pelvic discontinuity. Chirurgia Narządów Ruchu i Ortopedia Polska. 2019; 84(2): 56-60. doi: 10.31139/chnriop.2019.84.2.11

9. Ibrahim DA, Fernando ND. Classifications in brief: the Paprosky classification of femoral bone loss. Clin Orthop Relat Res. 2017; 475: 917-21. doi: 10.1007/s11999-016-5012-Z

10. Shon WY, Santhanam SS, Choi JW. Acetabular Reconstruction in Total Hip Arthroplasty. Hip Pelvis. 2016; 28(1): 1-14. doi: 10.5371/ hp.2016.28.1.1

11. Hsu CC, Hsu CH, Yen SH, Wang JW. Use of the Burch-Schneider cage and structural allografts in complex acetabular deficiency: 3- to 10year follow up. Kaohsiung J Med Sci. 2015; 31(10): 540-7. https://doi. org/10.1016/j.kjms.2015.08.001

12. Khuangsirikul S, Chotanaphuti T. Management of femoral bone loss in revision total hip arthroplasty. J Clin Orthop Trauma. 2020; 11(1): 29-32. doi: 10.1016/j.jcot.2019.12.004

13. Dipaola M, Wodajo F. M, 3D Printing in Orthopaedic Surgery. Elsevier, 2019.

14. Kaziunas, France A. Świat druku 3D, Przewodnik. Helion, 2014.

15. Siemiński P, Budzik G. Techniki przyrostowe. Druk 3D. Drukarki 3D. OWPW, 2015.

16. Sperring TR, Wintermeyer E, DeRogatis MJ, Di Cesare PE, Issack PS. Massive Acetabular Bone loss Reconstructed with a Custom Made Triflange Acetabular Component during Revision Hip Arthroplasty. Case Report. World J Surg Surgical Res. 2018; 1: 1073. 